\title{
Urgences
}

\section{Pauline Harvey, La ville aux gueux, Montréal, Éditions de la pleine lune, 1982, 256 p.}

\section{Renald Bérubé}

Numéro 14, août 1986

Corps et jouissances

URI : https://id.erudit.org/iderudit/025273ar

DOI : https://doi.org/10.7202/025273ar

Aller au sommaire du numéro

\section{Éditeur(s)}

Urgences

\section{ISSN}

0226-9554 (imprimé)

1927-3924 (numérique)

Découvrir la revue

Citer ce compte rendu

Bérubé, R. (1986). Compte rendu de [Pauline Harvey, La ville aux gueux, Montréal, Éditions de la pleine lune, 1982, 256 p.] Urgences, (14), 84-86. https://doi.org/10.7202/025273ar d'utilisation que vous pouvez consulter en ligne.

https://apropos.erudit.org/fr/usagers/politique-dutilisation/ 
écrit, cet humour de la lenteur: "[...] pour goûter comme pour les actes d'amour, si vous courez, pressés, abstenezvous, de grâce, la vitesse en ces deux matières débouche sur la tristesse ou se débande en regrets" (p. 165). Quant à la phrase de l'écrivain, elle est parfois attentive à ses effets - "Boire le poison ou avoir dans la bouche ce mot équivaut." (p. 101) - à sa courbe sonore ou à l'efficace du signifiant; cependant, justement parce que tout est affaire de langue, donc de goût et de langage, donc d'écriture, le propos philosophique concerte avec le poème - "Mon désir s'identifie à l'écrit, je n'existe qu'en langue." (p. 55) - avec cette gymnastique langagière que sollicite tout texte: "Sait-on qu'écrire exige l'habileté nerveuse et musculaire la plus fine?" ( $p$. 86). C'est sans doute pourquoi l'écriture s'adonne à cette nomination passionnée des choses, des substances, des goûts, des fibres: elle nous rend les données élémentaires du réel - "Le donné ressenti équivaut-il au dit?" (p. 102) -. et sous un apparent éclectisme culturel. elle parle de l'unité consubstantielle du monde.

Ainsi, en cette "certaine fin de siècle", peut-on encore tirer enseignement d'une philosophie qui ne dénie pas le sensualisme, d'une écriture qui agit conjointement dans la perception et l'expression. d'une morale des sens (et des significations) retrouvés? Michel Serres nous y convie comme en un recommencement contemporain du Banquet:

viens, je voudrais te léguer les choses sensibles perdues, le conciliabule du monde multiple et du corps nué. je te léguerai la finesse. goûis et parfums. la sapience et la sagacite, viens, et quand nous aurons construit la peau par pans. comme un habit. je te dirai. après. les vieilles ruines de ma langue. mon beau langage qui va mourir. issu directement de l'eau qui se chiffomme comme une soie. du peuplier dont la feuille sessile bruisse, douce voix des choses, viens dans les restes abandonnés des deux jardins pillés, oubliés, le jardin des sens détruit par le langage. le jardin de ma langue détruit par les codes. viens tant qu il est temps encore, je my etais mal pris, nous allons recommencer. (p. 112).

Les cinq sens: à lire comme directives. dans toutes les directions.

\section{Paul Chanel Malenfant} UQAR

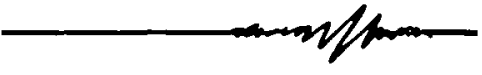

\section{Pauline Harvey: La ville aux gueux, Montréal, Éditions de la pleine lune, 1982, 256 pages.}

La ville aux gueux: roman merveilleux. c'est-à-dire des merveilles et des jeux. Des merveilles, comme dans Alice aux pays desdites (le chapitre XI de $L a$ ville, en particulier, sait justement recréer, mimétisme voulu, le nonsense carrollien), pays des étonnements et des prodiges. des anachronismes et des dépaysements, des merveilles donc wonderland. Des jeux. ceux de la scène comme de la politique, des mots et du langage - comme dans Alice au pays du langage de Marina Yaguello (Seuil. 1981) - jeux divers du mensonge et de la vérité, des apparences et des oripeaux, Homo ludens selon le titre du livre de J. Huizingua: jeu qui fait passer du chapitre XII au chapitre XIV dans un roman où le magique... joue un rôle important. La ville aux gueux. donc: roman ludique. des merveilles de l'imagination débridée (c'est-à-dire bien organisée. structurée).

Incipit oblige. commençons par le commencement

Trois pelenns veztus de haillons pesants s'en venaient d'une démarche claudicante 
sur une route ennejgee du pays de Fhartag.

- Croyez-vous quion va arriver à temps pour les vèpres? dit le plus grand, qui boitait un peu et s'appuyait sur une longue canne noueuse.

On était à la fin de la Renaissance sur cette route de Fhartag qui menait à la ville de Varthal et c'était important d'artiver pour les vêpres... (p. 9)

Les trois compères: Cécil, Lyly et Rozie arriveront à temps à Varthal _ et il faut voir (lire) comment, dans la suite de ce premier chapitre, ils s'inventent au fur et à mesure, pour les habitants de la ville et pour les lecteurs, une identité. Comme plus tard Rozie inventera l'Italie pour la Comtesse d'Alpenstock. Si bien que le trio, en ces lieux où règne le roi Arteur (Arthur + acteur?), se retrouve bientôt pourvoyeur de la scène thêâtrale. Cécil écrit les pièces déguisant pour la circonstance son style avonien en style néo-euphéiste (p. 81 sq.: suite à ce sujet au paragraphe suivant). Succès. Mais Cécil est bientôt démasqué par un écrivain jaloux. Riposte de Cécil par la scène même, danger d'émeute. Le théâtre, l'invention, aura le meilleur sur le politique répressif et à courte vue. D'autant plus que Varthal, plus que la Cour, est de fait gouvernée dans son imaginaire par les gens pauvres du quartier des ponts (p. 74 et 223). Déguisée en Barberousse des Pays-Bas. "trouvère en langue d'oil" (p. 192), l'enfant unique d'Arteur, la Princesse Smine, qui se fait appeler Prince Smine par "caprice féministe" (p. 12-13), refera à son tour et à sa manière, avec la complicité de Comédie, de Mississipi Free et autres gens des ponts et pour se venger de Cécil et des siens, la démarche du trio. Cécil et Smine finiront pourtant... N'essayons pas davantage de résumer les rebondissements multiples du roman, mais affirmons pourtant ceci en le soulignant: la Ville aux gueux, roman québécois de la Renaissance et du blues, roman des merveilles de l'imagination et des paradoxes du jeu, est aussi une fort belle réflexion sur la naissance des cultures, sur les rapports entre peuples, classes et cultures. Le blues existait déjà à la Renaissance. Sous d'autres formes - que deviennent alors ce que nous appelons les anachronismes?

Et nous voilà arrivés au paragraphe annoncé il y a quelques lignes déjà. II faut alors relire le passage suivant de $\mathrm{La}$ Ville:

-- Oui. dit Lyly. J'ai trouvé un vieux livre qui a l'air de résumer à peu près ce qu'ils ont fait à Varthal. Il sortit et revint bientôt avec un bouquin. Ils ont une théorie très précise quils ont appelèe eupheuisme. Leur style se caractérise par une affectation et un maniérisme qui leur paraît à eux le comble du raffinement. (p. 81).

Or il y eut bien, dans l'Angleterre élisabéthaine, un auteur appelé John Lyly (1554?-1606) qui publia aux alentours de la Noël de 1578, un roman de 1578 (la Ville commence quelques jours avant Noël), un roman intitulé Euphues. The Anatomy of Wit. Succès considérable, qui donna naissance à une suite Euphues and His England (1580)

L'Euphuisme (sans e médian) qui, précisément. peut se définir ainsi que dans la citation qui précède et sans que ce "raffinement" ait nécessairement un sens péjoratif, eut beaucoup d'adeptes; entre autres et par exemple. Thomas Lodge (1558-1625) qui publie Rosalynde or Euphues' Golden Legacie en 1590 , roman qui inspirera Comme il vous plaira (1600?) de Shakespeare. De fait, l'Avonie de Cécil, les difficultés de ce dernier avec un gros bourgeois de sa région natale (p. 35), son "ami avonien qui est librairie à Varthal" (p. 180) évoquent irrésistiblement des éléments que l'on retrouve dans la biographie de Sha. kespeare (Jean Paris, Shakespeare par lui-même, Paris, Seuil, 1954, p. 43 et 49): plus encore, l'influence de l'Italie, l'évocation des moralités (p. 91) et du goût du théâtre venu des auberges ( $p$. 67), de la situation des gens de théâtre (p. 171 et 187) et autres allusions multiples (peste. magie, etc.) nous ren- 
voient bien à la société élisabéthaine de la fin du XVIe siècle, à la naissance et à la montée du théâtre dit élisabéthain, théâtre populaire dans ses origines. Les anachronismes, alors, c'est aussi la façon qu'ont les siècles de se parler et de se répondre l'un à l'autre: la Renaissance, c'est aussi aujourd' hui et Hamlet, on le sait depuis Gurik, peut bien être le Prince du Québec. Et vive la transtextualité après (avec) l'imagination!

La ville aux gueux: roman néoeupheuiste selon la description même que ses personnages donnent de cette notion (p. 83-84)? Au jeu alors, jeu comme dans play within a play!

Renald Bérubé 